\title{
AN EIGENVALUE PROBLEM INVOLVING A FUNCTIONAL DIFFERENTIAL EQUATION ARISING IN A CELL GROWTH MODEL
}

\author{
BRUCE VAN BRUNT ${ }^{\bowtie 1}$ and M. VLIEG-HULSTMAN ${ }^{1}$
}

(Received 17 August, 2009; revised 8 September, 2010)

\begin{abstract}
We interpret a boundary-value problem arising in a cell growth model as a singular Sturm-Liouville problem that involves a functional differential equation of the pantograph type. We show that the probability density function of the cell growth model corresponds to the first eigenvalue and that there is a family of rapidly decaying eigenfunctions.
\end{abstract}

2000 Mathematics subject classification: primary 34K06; secondary 34K10.

Keywords and phrases: pantograph equation, cell growth model.

\section{Introduction}

This paper is concerned with solutions to functional differential equations of the form

$$
y^{\prime}(x)+b x^{n} y(x)=\lambda \alpha^{n} x^{n} y(\alpha x),
$$

subject to

$$
\lim _{x \rightarrow 0^{+}} y(x)=0, \quad \lim _{x \rightarrow \infty} y(x)=0,
$$

and the normalizing condition

$$
\int_{0}^{\infty} y(x) d x=1 .
$$

Here, $b>0, \alpha>1$ are constants, $n$ is a nonnegative integer, and $\lambda$ is a nonzero eigenvalue parameter. Equation (1.1) and conditions (1.2) and (1.3) form a first-order singular Sturm-Liouville problem, which, in this paper, we shall refer to simply as the boundary-value problem.

If $n=0$, then (1.1) is the pantograph equation. This equation has been studied extensively by several researchers (for example, $[2,7,8]$ ) owing to its wide range of applications, including light absorption in the Milky Way [1], current collection for an

\footnotetext{
${ }^{1}$ Institute of Fundamental Sciences, Mathematics, Massey University, Palmerston North, New Zealand; e-mail: b.vanbrunt@massey.ac.nz.

(C) Australian Mathematical Society 2010, Serial-fee code 1446-1811/2010 \$16.00
} 
electric train [3,9], probability theory [4], and more recently a cell growth model [5]. Most of these studies focused on the asymptotic behaviour of solutions as $x \rightarrow \infty$ for initial-value problems; however, the cell growth model brought boundary-value problems to the fore. The cell growth model of Hall and Wake [5] is based on the equation

$$
\frac{\partial}{\partial t} n(x, t)+\frac{\partial}{\partial x}(g(x, t) n(x, t))=-b(x, t) n(x, t)+\alpha^{2} b(\alpha x, t) n(\alpha x, t),
$$

where $n$ is the number density of cells of size $x$ (as measured by DNA content) at time $t$, and $b$ is the rate at which cells of size $x$ are dividing and creating $\alpha$ new cells of size $x / \alpha$. The function $g$ is the growth rate of the cells. Under the assumption that the functions $b$ and $g$ depend solely on $x$, Hall and Wake looked for separable solutions $n(x, t)=N(t) y(x)$. These were called steady size distribution (SSD) solutions. Here, $y$ is a probability density function for the size of a cell. The separation process leads to the pantograph equation

$$
y^{\prime}(x)+a y(x)=a \alpha y(\alpha x),
$$

where $a=\alpha b / g$. A second-order version of the problem was studied in [10].

There is a paucity of information about linear functional differential equations with variable coefficients. In a second paper on cell growth, however, Hall and Wake [6] studied the boundary-value problem for the case where the growth rate is assumed to be linear and the division rate is of the form $b(x)=b x^{n}$, where $b$ is a constant. These assumptions lead to an equation of the form (1.1). Their study included the more general case where $n$ is not necessarily an integer.

In this paper we show that the boundary-value problem leads to a family of eigenvalues and corresponding eigenfunctions. The first eigenfunction is the probability density function from the cell growth model. The problem is solved using Mellin transforms. We recover the results given by Hall and Wake for the first eigenfunction and then look at the higher eigenfunctions.

\section{A Mellin transform solution}

Equation (1.1) can be transformed into the pantograph equation with constant coefficients. The Dirichlet series solution for the pantograph equation can then be exploited to find a solution to the problem. This approach was used by Hall and Wake [6] for the case $\lambda=b \alpha$, but it can be adapted for more general values. Alternatively, one can apply the Mellin transform to (1.1) and solve the problem directly. In this section, we formulate and solve the equation for the Mellin transform and its inverse. We use a technique of factoring the transform into two parts, one of which does not depend on the functional parameter $\alpha$. It may be possible that this approach can be adapted to more general functional equations; however, the solution of the transform equation might prove formidable. The Mellin transform approach has the advantage that it highlights eigenvalues and the role of solutions to 
the homogeneous equation

$$
y^{\prime}(x)+b x^{n} y(x)=0 .
$$

It also indicates possible sources for nonuniqueness.

Recall that the Mellin transform of a function $f$ is

$$
M[f, s]=\int_{0}^{\infty} x^{s-1} f(x) d x .
$$

We first establish conditions for a fundamental strip in the $s$-plane.

LEMMA 2.1. Let y be a solution to (1.1) such that $y$ is differentiable in some interval $[0, a], a>0$ and $y(0)=0$. Then $y$ has derivatives of all orders in $[0, a]$ and, for all $k \in \mathbb{N}, y^{(k)}(0)=0$; consequently,

$$
\lim _{x \rightarrow 0^{+}} \frac{y(x)}{x^{k}}=0,
$$

for all $k \in \mathbb{N}$.

PROOF. Equation (1.1) implies that

$$
y^{\prime}(x)=x^{n}\left(\lambda \alpha^{n} y(\alpha x)-b y(x)\right),
$$

so that $y^{\prime}(0)=0$. Since $y$ is differentiable, we can differentiate the above expression to get

$$
y^{\prime \prime}(x)=n x^{n-1}\left(\lambda \alpha^{n} y(\alpha x)-b y(x)\right)+x^{n}\left(\lambda \alpha^{n+1} y^{\prime}(\alpha x)-b y^{\prime}(x)\right) .
$$

Since $y$ and $y^{\prime}$ both vanish at $x=0$, the right-hand side of the above expression is also zero when $x=0$ even if $n=1$. We thus see that $y^{\prime \prime}(0)=0$. It is clear that we can continue this process any number of times to show that $y$ has derivatives of all orders and that $y^{(k)}(0)=0$ for all $k \in \mathbb{N}$. Equation (2.2) follows from L'Hôpital's rule.

THEOREM 2.2. Let y be a solution to the boundary-value problem such that

$$
\int_{0}^{\infty} x^{n+1}|y(x)| d x<\infty .
$$

Then the fundamental strip for the Mellin transform of $y$ includes the interval $(-\infty, n+2]$.

PROOF. Inequality (2.3) and the comparison test show that $M[y, s]$ is defined for all $s$ such that $1 \leq s \leq n+2$. Suppose that $s<1$. Then $s-1<0$, but Lemma 2.1 implies that $x^{s-1} y(x) \rightarrow 0$ as $x \rightarrow 0^{+}$. Hence $x^{s-1} y(x)$ is continuous on $[0,1]$ and the integral

$$
\int_{0}^{1} x^{s-1} y(x) d x
$$


is thus well defined. The integral

$$
\int_{1}^{\infty} x^{s-1} y(x) d x
$$

converges by the comparison test and therefore $M[y, s]$ is defined for all $s<1$.

We restrict our attention to functions $y$ that satisfy condition (2.3). In addition, we assume that

$$
\lim _{x \rightarrow \infty} x^{n+1} y(x)=0 .
$$

The above conditions imply that

$$
M\left[y^{\prime}, s\right]=\left.x^{s-1} y(x)\right|_{0} ^{\infty}-(s-1) \int_{0}^{\infty} x^{s-2} y(x) d x=-(s-1) M[y, s-1] .
$$

Taking the Mellin transform of both sides of (1.1) gives

$$
-(s-1) M(s-1)+b M(s+n)=\frac{\lambda}{\alpha^{s}} M(s+n),
$$

where, for succinctness, we use $M(s)=M[y, s]$. Condition (1.3) implies that

$$
M(1)=1 .
$$

We seek solutions to (2.5) of the form

$$
M(s)=K F(s) Q(s),
$$

where $K$ is a nonzero constant that will be used later for normalization and $F$ corresponds to the Mellin transform equation for the homogeneous equation (2.1). We thus have

$$
-(s-1) F(s-1)+b F(s+n)=0,
$$

and since we can solve (2.1) by elementary means, we know that a solution to (2.8) is

$$
F(s)=\frac{1}{n+1}\left(\frac{b}{n+1}\right)^{-s /(n+1)} \Gamma\left(\frac{s}{n+1}\right) .
$$

The second factor $Q$ contains all the influence of the functional term. Substituting (2.7) into (2.5) gives

$$
-(s-1) F(s-1) Q(s-1)+\left(b-\frac{\lambda}{\alpha^{s}}\right) F(s+n) Q(s+n)=0,
$$

and, using (2.8), we have that

$$
Q(s-1)=\left(1-\frac{\lambda}{b \alpha^{s}}\right) Q(s+n)
$$


which has a solution

$$
Q(s)=\prod_{k=0}^{\infty}\left(1-\frac{\lambda}{b \alpha^{k(n+1)+1+s}}\right) .
$$

One solution to (2.5) is therefore

$$
M(s)=\frac{K}{n+1}\left(\frac{b}{n+1}\right)^{-s /(n+1)} \Gamma\left(\frac{s}{n+1}\right) \prod_{k=0}^{\infty}\left(1-\frac{\lambda}{b \alpha^{k(n+1)+1+s}}\right) .
$$

Note that $M$ is defined for all $s>0$, and that

$$
M(1)=\frac{K}{n+1}\left(\frac{b}{n+1}\right)^{-1 /(n+1)} \Gamma\left(\frac{1}{n+1}\right) \prod_{k=0}^{\infty}\left(1-\frac{\lambda}{b \alpha^{k(n+1)+2}}\right),
$$

so that there is a $K$ that satisfies condition (2.6) for $Q(1) \neq 0$, that is, provided that

$$
\lambda \neq b \alpha^{k(n+1)+2}
$$

for any nonnegative integer $k$.

Integrating (1.1) from 0 to $\infty$ gives

$$
\left(b-\frac{\lambda}{\alpha}\right) M(n+1)=0
$$

hence, either $\lambda=b \alpha$ or $M(n+1)=0$. In any case, the product $Q$ vanishes at $s=0$. The eigenvalues are therefore

$$
\lambda_{m}=b \alpha^{m(n+1)+1}
$$

where $m$ is a nonnegative integer. The spectrum $\left\{\lambda_{m}\right\}$ of eigenvalues for the class of solutions given by $(2.10)$ is discrete. If $k(n+1)+2=m(n+1)+1$, then $m-k=$ $1 /(n+1)$, and for $n>0$ there are no integers $m, k$ that satisfy this relation. Every eigenvalue therefore satisfies condition (2.11) and the normalizing constant from (2.6) is

$$
K_{m}=(n+1)\left(\frac{b}{n+1}\right)^{1 /(n+1)}\left(\Gamma\left(\frac{1}{n+1}\right)\right)^{-1} \prod_{k=0}^{\infty}\left(1-\frac{1}{b \alpha^{(k-m)(n+1)+1}}\right)^{-1}
$$

Note that the gamma function in $M(s)$ has simple poles at $s=-\ell(n+1)$, where $\ell$ is a nonnegative integer. If $\lambda \in\left\{\lambda_{m}\right\}$, then $Q$ has a simple zero at $s=-\ell(n+1)$. The choice of an eigenvalue thus ensures that $M$ is defined for all $s \leq 0$. Alternatively, Theorem 2.2 implies that $\lambda \in\left\{\lambda_{m}\right\}$. The spectrum thus corresponds to the values of $\lambda$ that make the Mellin transform well defined for $s \leq 0$.

The separable form of the Mellin transform makes it tractable to invert. The inversion is based on the simple observation that if $G(s)$ is the Mellin transform of $g(x)$, then $\alpha^{-k s} G(s)$ is the Mellin transform of $g\left(\alpha^{k} x\right)$. The product $Q(s)$ can be 
expressed as a power series in $\alpha^{-s}$, by use of the Euler identity,

$$
\prod_{k=0}^{\infty}\left(1+z q^{k}\right)=1+\sum_{k=1}^{\infty} \frac{q^{k(k-1) / 2} z^{k}}{\prod_{j=1}^{k}\left(1-q^{j}\right)},
$$

which is valid for $|q|<1$ and $z \in \mathbb{C}$. Specifically, let $q=\alpha^{-(n+1)}$ and $z=$ $-\lambda /\left(b \alpha^{s+1}\right)$. Then,

$$
Q(s)=\prod_{k=0}^{\infty}\left(1+z q^{k}\right)=1+\sum_{k=1}^{\infty} \frac{p_{k}(\lambda)}{\alpha^{k s}}
$$

where

$$
p_{k}(\lambda)=\frac{(-1)^{k}(\lambda /(b \alpha))^{k}}{\alpha^{(n+1) k(k-1) / 2} \prod_{j=1}^{k}\left(1-\alpha^{-(n+1) j}\right)} .
$$

The Mellin transform (2.7) can thus be written

$$
M(s)=K\left(F(s)+\sum_{k=1}^{\infty} p_{k}(\lambda) \frac{F(s)}{\alpha^{k s}}\right) .
$$

The infinite product in the Euler identity is uniformly convergent with respect to $z$ in any compact subset of the complex plane, and this indicates that the Mellin transform can be inverted term by term. The inverse transform of $F$ is

$$
f(x)=e^{-b x^{n+1} /(n+1)} \text {; }
$$

therefore, solutions to the boundary-value problem are given by

$$
y_{m}(x)=K_{m}\left(e^{-b x^{n+1} /(n+1)}+\sum_{k=1}^{\infty} p_{k}\left(\lambda_{m}\right) e^{-b \alpha^{k(n+1)} x^{n+1} /(n+1)}\right) .
$$

The Euler identity implies that

$$
1+\sum_{k=1}^{\infty} p_{k}\left(\lambda_{m}\right)=0
$$

so that membership of $\lambda$ in the spectrum ensures that $y(0)=0$.

\section{Uniqueness}

The analysis of the previous section established that the boundary-value problem has solutions when $\lambda$ is in the spectrum. The restriction on the class of functions for the Mellin transform analysis amounts to a strong decay condition. Certainly the Dirichlet series solutions (2.15) decay rapidly as $x \rightarrow \infty$, but it may be that there are other solutions. We know from the analysis of Kato and McLeod [8] that the pantograph equation has an infinite number of solutions for a given initial value at $x=0$. We also know from the work of Hall and Wake [5] that the boundary-value problem has a unique solution for the case $n=0$. 
A notable feature of (2.5) is that, given $M(1)$, the equation determines only $M(n(j-1)+j)$ for $j \in \mathbb{N}$. For instance, $M(2)$ is not determined by this equation when $n>0$. The absence of information about $M(2)$ and other Mellin transform values signals that the Dirichlet series solutions may not be the only solutions even with the assumption of strong decay. Certainly, the Mellin transform solution (2.10) is not unique since $M$ can be multiplied by any nonzero function $P$ that satisfies $P(s)=P(s+n+1)$. For example,

$$
\hat{M}(s)=K \frac{F(s) Q(s)}{\sin (2 \pi s /(n+1))}
$$

is also a solution to (2.5). Functions such as $P$ may not lead to solutions of the boundary-value problem, but there is latitude for nonuniqueness. In this section we show that under certain rapid decay conditions, the Dirichlet series solutions are the only solutions.

The first eigenfunction $y_{0}$ is important in the application to the cell growth model. This function should represent a probability density function and therefore the condition $y(x) \geq 0$ for all $x>0$ is required by the model. Hall and Wake [6] showed that $y_{0}$ is a probability density function indirectly by mapping it back to the pantograph equation. We will show this directly from the equation along with a uniqueness result. The direct approach also provides a pathway to establish uniqueness results for the higher eigenfunctions.

LEMMA 3.1. Let $v$ be a differentiable function on $[0, \infty)$ such that $v(x) \rightarrow 0$ as $x \rightarrow \infty$, and

$$
v^{\prime}(x)=b x^{n}(v(\alpha x)-v(x)) .
$$

If $v^{\prime}\left(x_{0}\right)=0$ for some $x_{0} \in(0, \infty)$, then $v(x)=0$ for all $x \in[0, \infty)$.

PROOF. We show first that $v$ cannot have local extrema in $(0, \infty)$. Suppose that $v$ has a positive local maximum at $M_{1} \in(0, \infty)$. Equation (3.1) implies that $v\left(M_{1}\right)=$ $v\left(\alpha M_{1}\right)$, and since $v(x) \rightarrow 0$ as $x \rightarrow \infty$ there must exist another local maximum at some point $M_{2} \geq \alpha M_{1}$ at which $v\left(M_{2}\right) \geq v\left(M_{1}\right)$. We can use the same argument to establish the existence of another local maximum $M_{3} \geq \alpha M_{2}$ at which $v\left(M_{3}\right) \geq$ $v\left(M_{2}\right)$, and it is clear that this argument can be repeated ad infinitum to show that there must be a sequence of local maxima $\left\{M_{j}\right\}$ such that $M_{j} \rightarrow \infty$ as $j \rightarrow \infty$ and, for all $j \in \mathbb{N}$,

$$
v\left(M_{j}\right) \geq v\left(M_{1}\right)>0 .
$$

The above inequality, however, contradicts the assumption that $v(x) \rightarrow 0$ as $x \rightarrow \infty$; therefore, $v$ cannot have a positive local maximum. This conclusion can also be applied to the function $-v$ so that $v$ cannot have a negative local minimum. If $v$ has a negative local maximum then the condition $v(x) \rightarrow 0$ as $x \rightarrow \infty$ implies that it must have a negative local minimum. Similarly, if $v$ has a positive local minimum, it must have a positive local maximum. Therefore, $v$ cannot have local extrema in $(0, \infty)$. 
Suppose that $v^{\prime}\left(x_{0}\right)=0$ for some $x_{0} \in(0, \infty)$. Then (3.1) implies that $v\left(x_{0}\right)=$ $v\left(\alpha x_{0}\right)$, and since $v$ cannot have local extrema it follows that $v(x)=v\left(x_{0}\right)$ for all $x \in\left[x_{0}, \alpha x_{0}\right]$. Therefore $v^{\prime}(x)=0$ for all $x \in\left[x_{0}, \alpha x_{0}\right]$. Evidently, this argument can be repeated to show that $\nu^{\prime}(x)=0$ for all $x \in\left[\alpha x_{0}, \alpha^{2} x_{0}\right]$. The argument can be repeated any number of times and this implies that $\nu^{\prime}(x)=0$ for all $x \in\left[x_{0}, \infty\right)$. Since $v(x) \rightarrow 0$ as $x \rightarrow \infty, v(x)=0$ for all $x \in\left[x_{0}, \infty\right)$, and (3.1) implies that

$$
v^{\prime}(x)+b x^{n} v(x)=0,
$$

for $x \in\left[x_{0} / \alpha, \infty\right)$. The above relation is an ordinary differential equation for $v$ that must satisfy the initial condition $v\left(x_{0}\right)=0$. Picard's theorem shows that the unique solution to this problem is the trivial solution; hence, $v(x)=0$ for all $x \in\left[x_{0} / \alpha, \infty\right)$. This argument can be repeated $j$ times to show that $v(x)=0$ for all $x \in\left[x_{0} / \alpha^{j}, \infty\right)$, and this implies that $v(x)=0$ for all $x>0$. The continuity of $v$ shows that $v(0)=0$.

THEOREM 3.2 (The first eigenfunction). The function $y_{0}$ defined by (2.15) is a solution to the boundary-value problem with $\lambda=b \alpha$. This solution is unique among functions $y$ such that

$$
\int_{0}^{\infty} x^{n} y(x) d x<\infty .
$$

Moreover, $y_{0}(x)>0$ for all $x>0$.

PROOF. It was established in the previous section that $y_{0}$ is a solution to the boundaryvalue problem. Suppose that $y$ and $w$ satisfy condition (3.2) and are solutions to the boundary-value problem, and let $z=y-w$. Then $z$ satisfies condition (3.2), the equation

$$
z^{\prime}(x)+b x^{n} z(x)=\lambda \alpha^{n} x^{n} z(\alpha x)
$$

and also the relation

$$
\int_{0}^{\infty} z(x) d x=0 .
$$

Since $z$ satisfies condition (3.2), the function

$$
\delta(x)=\int_{x}^{\infty} \xi^{n} z(\xi) d \xi
$$

is defined for all $x \geq 0$. Integrating (3.3) from 0 to $\infty$ yields

$$
-z(x)=b(\delta(\alpha x)-\delta(x))
$$

and since

$$
\delta^{\prime}(x)=-x^{n} z(x)
$$

we have

$$
\delta^{\prime}(x)=b x^{n}(\delta(\alpha x)-\delta(x)) .
$$

Equation (3.4) implies that either $z$ is identically zero or $z$ must change sign in $(0, \infty)$; consequently, there is an $x_{0} \in(0, \infty)$ such that $z\left(x_{0}\right)=0$. Equation (3.5) implies 
that $\delta^{\prime}\left(x_{0}\right)=0$. Lemma 3.1 implies that $\delta(x)=0$ and hence $\delta^{\prime}(x)=0$ for all $x \geq 0$. Equation (3.5) shows that $z(x)=0$ for all $x \geq 0$, and the solution $y_{0}$ is therefore unique.

The above argument can also be used to show that $y_{0}$ is positive. We can replace $z$ with $y_{0}$ in the above arguments. Condition (1.3), however, does not require $y_{0}$ to change sign. Indeed, the above arguments show that if $y_{0}$ did change sign, then $y_{0}$ would be identically zero. Condition (1.3) shows that $y_{0}$ must be positive somewhere in $(0, \infty)$, and since it cannot change sign we conclude that $y_{0}(x)>0$ for all $x>0$.

The uniqueness of the higher eigenfunctions can be established in a manner similar to that used for the first eigenfunction. The decay condition, however, becomes more complicated. Let $\left\{\delta_{j}\right\}$ be a sequence of operators defined by

$$
\begin{aligned}
& \delta_{0} y(x)=y(x) \\
& \delta_{j} y(x)=\int_{x}^{\infty} \xi^{n} \delta_{j-1} y(\xi) d \xi,
\end{aligned}
$$

where $j \in \mathbb{N}$. Let $\mathcal{R}_{j}$ denote the set of functions $y \in C^{0}[0, \infty)$ such that

$$
\lim _{x \rightarrow \infty} x^{n} \delta_{k} y(x)=0
$$

and

$$
\delta_{k} y(0)<\infty
$$

for all $k=1,2, \ldots, j$. The Dirichlet series solutions $\left\{y_{m}\right\}$ are evidently in $\mathcal{R}_{j}$ for any $j \in \mathbb{N}$. Theorem 3.2 shows that $y_{0}$ is the unique solution to the boundary-value problem in $\mathcal{R}_{1}$.

The usefulness of the operator $\delta_{j}$ is that it can be used to map (1.1) back to (1.1) with a lower eigenvalue parameter.

LEMMA 3.3. Let $z_{m} \in \mathcal{R}_{m+1}$ be a solution to (1.1) with $\lambda=\lambda_{m}$. Then for $j=$ $0,1, \ldots, m$,

$$
\delta_{j+1}^{\prime}(x)=b x^{n}\left(\alpha^{(m-j)(n+1)} \delta_{j+1}(\alpha x)-\delta_{j+1}(x)\right),
$$

where $\delta_{j}(x)=\delta_{j} z_{m}(x)$.

PROOF. Equation (1.1) implies that

$$
z_{m}^{\prime}(x)=x^{n}\left(\lambda_{m} \alpha^{n} z_{m}(\alpha x)-b z_{m}(x)\right)=b x^{n}\left(\alpha^{(m+1)(n+1)} z_{m}(\alpha x)-z_{m}(x)\right),
$$

and integrating from $x$ to $\infty$ gives

$$
-z_{m}(x)=b\left(\alpha^{m(n+1)} \delta_{1}(\alpha x)-\delta_{1}(x)\right)
$$

For any $0 \leq j \leq m$,

$$
\delta_{j+1}^{\prime}(x)=-x^{n} \delta_{j}(x)
$$


hence,

$$
\delta_{1}^{\prime}(x)=b x^{n}\left(\alpha^{m(n+1)} \delta_{1}(\alpha x)-\delta_{1}(x)\right) .
$$

Given that $z_{m} \in \mathcal{R}_{m+1}$, the functions $\delta_{j}$ are well defined and $x^{n} \delta_{j}(x) \rightarrow \infty$ as $x \rightarrow \infty$ for $0 \leq j \leq m+1$. We can thus repeat the argument up to $m$ times, so that (3.8) holds for $0 \leq j \leq m$.

THEOREM 3.4 (Higher eigenfunctions). The function $y_{m}$ defined by (2.15) is a solution to the boundary-value problem with $\lambda=\lambda_{m}$. This solution is unique among functions in $\mathcal{R}_{m+1}$.

PROOF. Let $w_{m} \in \mathcal{R}_{m+1}$ be another solution to the boundary-value problem and let $z_{m}=y_{m}-w_{m}$. Then $z_{m} \in \mathcal{R}_{m+1}, z_{m}$ satisfies (1.1), and

$$
\int_{0}^{\infty} z_{m}(x) d x=0 .
$$

The function $z_{m}$ satisfies the conditions of Lemma 3.3; therefore,

$$
\delta_{m+1}^{\prime}(x)=b x^{n}\left(\delta_{m+1}(\alpha x)-\delta_{m+1}(x)\right) .
$$

We will show that $\delta_{m+1}^{\prime}\left(x_{0}\right)=0$ for some $x_{0}>0$ and apply Lemma 3.1 to (3.12).

Equation (3.9) implies that

$$
-\int_{0}^{\infty} z_{m}(x) d x=b\left(\alpha^{m(n+1)-1}-1\right) \int_{0}^{\infty} \delta_{1}(x) d x
$$

therefore, by (3.11),

$$
\left(\alpha^{m(n+1)-1}-1\right) \int_{0}^{\infty} \delta_{1}(x) d x=0 .
$$

Since $\alpha>1$, the above equation implies that

$$
\int_{0}^{\infty} \delta_{1}(x) d x=0
$$

Repeating this argument, using Equations (3.8) and (3.10) with $j=1$, gives

$$
-\int_{0}^{\infty} \delta_{1}(x) d x=b\left(\alpha^{(m-1)(n+1)-1}-1\right) \int_{0}^{\infty} \delta_{2}(x) d x
$$

hence,

$$
\int_{0}^{\infty} \delta_{2}(x) d x=0
$$

The process can be repeated to show that

$$
\int_{0}^{\infty} \delta_{j}(x) d x=0
$$

for $0 \leq j \leq m$. For $j=m$ the equation above implies that there is an $x_{0}>0$ such that $\delta_{m}\left(x_{0}\right)=0$ and hence $\delta_{m+1}^{\prime}\left(x_{0}\right)=0$. Lemma 3.1 shows that $\delta_{m+1}(x)=0$ for all $x \geq 0$. 
Equation (3.10) therefore gives $\delta_{m}^{\prime}(x)=0$ and since $\delta_{m}\left(x_{0}\right)=0, \delta_{m}(x)=0$ for all $x \geq 0$. We can continue this argument back to show that $\delta_{j}(x)=0$ for $0 \leq j \leq m$ and $x \geq 0$. In particular, $z_{m}(x)=0$ for all $x \geq 0$. The solution is thus unique in $\mathcal{R}_{m+1}$.

\section{Conclusions}

The cell growth model posed by Hall and Wake $[5,6]$ has a rich underlying structure as a singular Sturm-Liouville problem that involves a first-order functional equation. In this paper we have shown that the probability density function of the cell growth model arises naturally as the first eigenvalue of the problem. The requirement that $y$ be a p.d.f. solution leads to this eigenvalue.

The role of the higher eigenvalues and more detailed properties of the higher eigenfunctions remain to be explored. The uniqueness results presented here require strong decay conditions: it may be possible to relax them. The original cell growth problem concerns the first eigenvalue, and the problem makes sense for any function that is integrable on $[0, \infty)$. Consequently, there is the possibility of a solution $y$ to the boundary-value problem that is integrable on $[0, \infty)$ but decaying slowly enough that $x^{n} y$ is not integrable. The uniqueness of the solution asserted in Hall and Wake [6] relies on the proof for the case $n=0$. The transformation they used to map the $n>0$ case to the $n=0$ case, however, requires implicitly that $x^{n} y$ is integrable in the uniqueness proof.

For the higher eigenfunctions, the integrability of $x^{n} y$ is needed, but the condition $y \in \mathcal{R}_{m+1}$ is somewhat stronger. There is consequently a window through which other solutions such that $x^{n} y$ is integrable but $y \notin \mathcal{R}_{m+1}$ might exist.

\section{References}

[1] V. A. Ambartsumian, "On the fluctuation of brightness of the Milky Way", Dokl. Akad. Nauk USSR 44 (1944) 223-226.

[2] G. Derfel and A. Iserles, "The pantograph equation in the complex plane", J. Math. Anal. Appl. 213 (1997) 117-132.

[3] L. Fox, D. F. Mayers, J. R. Ockendon and A. B. Tayler, "On a functional differential equation", J. Inst. Math. Appl. 8 (1971) 271-307.

[4] D. P. Gaver, “An absorption probablilty problem”, J. Math. Anal. Appl. 9 (1964) 384-393.

[5] A. J. Hall and G. C. Wake, "A functional differential equation arising in the modelling of cellgrowth", J. Aust. Math. Soc. Ser. B 30 (1989) 424-435.

[6] A. J. Hall and G. C. Wake, "Functional differential equations determining steady size distributions for populations of cells growing exponentially", J. Aust. Math. Soc. Ser. B 31 (1990) 434-453.

[7] A. Iserles, "On the generalized pantograph functional-differential equation", European J. Appl. Math. 4 (1993) 1-38.

[8] T. Kato and J. B. McLeod, "The functional differential equation $y^{\prime}(x)=a y(\lambda x)+b y(x)$ ", Bull. Amer. Math. Soc. 77 (1971) 891-937.

[9] J. R. Ockendon and A. B. Tayler, "The dynamics of a current collection system for an electric locomotive", Proc. R. Soc. Lond. A 322 (1971) 447-468.

[10] B. van-Brunt, G. C. Wake and H. K. Kim, "On a singular Sturm-Liouville problem involving an advanced functional differential equation”, European J. Appl. Math. 12 (2001) 625-644. 